\title{
Using Network Science to Support Design Research \\ From Counting to Connecting
}

Parraguez Ruiz, Pedro; Maier, Anja

\author{
Published in: \\ Experimental Design Research
}

Link to article, DOI:

10.1007/978-3-319-33781-4_9

Publication date:

2016

Document Version

Peer reviewed version

Link back to DTU Orbit

Citation (APA):

Parraguez Ruiz, P., \& Maier, A. (2016). Using Network Science to Support Design Research: From Counting to Connecting. In P. Cash, T. Stankovi, \& M. Storga (Eds.), Experimental Design Research: Approaches, Perspectives, Applications (pp. 153-172). Springer. https://doi.org/10.1007/978-3-319-33781-4 9

\section{General rights}

Copyright and moral rights for the publications made accessible in the public portal are retained by the authors and/or other copyright owners and it is a condition of accessing publications that users recognise and abide by the legal requirements associated with these rights.

- Users may download and print one copy of any publication from the public portal for the purpose of private study or research.

- You may not further distribute the material or use it for any profit-making activity or commercial gain

- You may freely distribute the URL identifying the publication in the public portal 


\title{
Using Network Science to Support Design Research: From Counting to Connecting
}

\author{
Pedro Parraguez and Anja Maier
}

\begin{abstract}
A network-based perspective on designing permits research on the complexity of product, process, and people interactions. Strengthened by the lat- est advances in information technologies and accessibility of data, a network- based perspective and use of appropriate network analysis metrics, theories, and tools allow us to explore new data-driven research approaches in design. These approaches allow us to move from counting to connecting, meaning to explicitly link disconnected pieces of data, information, and knowledge, and thus to answer far-reaching research questions with strong industrial and societal impact. This chapter contributes to the use of network science in empirical studies of design organisations. It focuses on introducing a network-based perspective on the design process and in particular on making use of network science to support design research and practice. The main contribution of this chapter is an overview of the methodological challenges and core decision points when embarking on networkbased design research, namely defining the overall research purpose and selecting network features. We furthermore highlight the potential for using archival data, the opportunities for navigating different levels of the design process that network analysis permits, what we here call zooming in and out, and the use of network visualisations. We illustrate the main points with a case from our own research on engineering communication networks. In this case, we have used more than three years of archival data, including design activity logs and work-related email exchanges from a recently completed large-scale engineering systems project of designing and developing a renewable power plant.
\end{abstract}

Keywords Network Analysis • Complexity • Design Process 


\subsection{Introduction: A Network-Based Perspective on the Engineering Design Process}

With the increased availability of empirical data and advanced analytical methods to acquire and analyse such data, experimental and observational design research has grown in volume and importance. This has meant that bridging and interpret- ing results obtained from different methodological approaches (e.g. qualitative and quantitative) and levels of analysis (from human behaviour to industrial ecosys- tems) is a pressing need in order to strengthen the scientific development of our discipline. Furthermore, the growing socio-technical complexity of the design pro- cess in engineering systems has led researchers increasingly to adopt a systemic view for studying design. One question that has emerged in this context is, how can we navigate and integrate results obtained at different levels of analysis and with different methodological approaches? Or in other words, how can we go from counting fragmented empirical findings to connecting and integrating them?

One way of answering this question is through a networked perspective of the design process; a perspective focused on how the myriad elements that play a role in the collective act of designing are connected. Following such a perspective, the design process can be modelled as a network of interactions between and within, for example, design engineers, project stakeholders, design activities, or product components. It is through this network-based perspective of design that we can go from counting to connecting and explicitly link otherwise disconnected data fragments. As a result of applying this networked perspective, we enable the generation of new insights about the mechanisms driving and affecting the design process (Parraguez2015).

However, the path from a research mode focused on counting, embodied in the form of tabular data and charts, to one focused on connecting, embodied in the form of relational data and network graphs, is not simple. Such a transition requires means for data acquisition, analysis, and interpretation, which until recently were new and mostly unexplored territory for the design research community. Some of the challenges of this transition include integrating, analysing, and visualising the vast amount of interactions between and within the process, organisation, and prod- uct domains, as well as navigating and integrating different levels of analysis. The objective of this chapter is to help translate the (growing) apparatus of methods and tools generated by the multiorigin and multidisciplinary field of network science for design researchers. The key result is an empirically grounded reflection about, and a guide to, important decision points regarding the selection of network fea- tures relevant for all those using network science in their design research studies. 


\subsubsection{Network Science and Its Application in Design Research}

Network science allows for integrative and multilevel analyses that explicitly consider interaction effects and non-linear relations between inputs and outputs. Such nonlinearity and interconnectedness is a core characteristic of complex systems (e.g. Strogatz 2001). As a result, network representations of systems in all kinds of fields have allowed us to gain access to new and valuable practical and theoretical insights. Insights that would have been otherwise out of reach hidden underneath a wealth of disconnected data pieces.

Due to the strengths and wide applicability of network-based approaches, researchers are increasingly modelling and analysing complex systems as networks. For example, network science principles and tools have been used to understand a variety of biological, social, technical, and socio-technical sys- tems and, in particular, the relation between structure and behaviour (Albert and Barabási 2002; Newman 2003). Furthermore, there is growing evidence about the existence of network properties common to a range of different complex systems with direct effect on the behaviour and performance of those systems (e.g. Ahn et al. 2010 and Braha in this book).

Consequently, the impact of the emergent science of networks is rapidly spreading through different fields and application areas, ranging from the study of intricate chains of protein-protein interactions to large-scale social networks that include millions of individuals (e.g. Vespignani 2009; Christakis and Fowler 2011). Hence, we have arrived at a point where understanding how a complex structure of interactions can generate useful (or harmful) behaviours has become crucial to managing the complexity of design, production, and management of human-made engineered systems (Calvano and John 2004; Storga et al. 2013).

Network studies of complex systems are not unknown in the context of design research, dating back to pioneering works of authors such as Simon (1962), Allen (1977), and Steward (1981). However, design research has not yet reached the maturity that fields such as computer science, physics, and sociology have, where there is a longer tradition and a stream of theoretical and methodological contributions to network science. It is for this reason that a grounded and contextualised support for the future use of network science in engineering design seems timely and appropriate.

Although the use and development of network science in design research are still far from mature compared to other research fields, in recent years we have seen an increase in the use of network analysis to support both theory building and theory testing while also enriching design management practice. Examples of this include researchers that have modelled and studied the architectures of process, organisational, and product domains as networks (Eppinger and Browning 2012; Eppinger and Salminen 2001). In the process domain, different variants of activity networks in matrix form (e.g. the Design Structure Matrix) and graph form have been applied to understand and analyse information dependencies between activities to optimise the logical sequence of activities and the impact of the process architecture on variables such as cost and time (Browning and Eppinger 2002; Steward 1981). In the organisational domain, traditional social network analysis with roots in sociology and organisational studies have influenced the analysis of networks of design engineers, focusing on aspects such as formal and informal relationships and communication exchanges (e.g. Allen 1977; Maier et al. 2008), creative interactions (e.g. Sosa 2010), 
and the analysis of organisational roles (e.g. Sonnenwald 1996). Finally, in the product domain, the interconnected architecture of components has also been analysed (Baldwin et al. 2013; Sosa et al. 2007) to explore issues such as product quality (e.g. Gokpinar et al. 2010; Sosa et al. 2011), the characterisation of modularity, and other complex product architectures (e.g. Sharman and Yassine 2004).

Further, a diverse set of network methods (Kreimeyer and Lindemann 2011; Lindemann et al. 2009) have been used to model aspects such as the temporal evolution of information across the design and development stages of an energy plant (Parraguez 2015); to explore how information flows through design activities and is exchanged between designers in the organisation (e.g. Batallas and Yassine 2006; Parraguez et al. 2015a); or to analyse the propagation of changes and errors in the design process (e.g. Braha and Bar-Yam 2007; Giffin et al. 2009; Wynn et al. 2014). While this recent body of research has advanced our theoretical understanding and analytical methods to analyse networks in a design context, it has also highlighted the need for a more cohesive, systematic, and reflective revision of the many network features that have been and can be analysed, as well as the methodological steps followed in their analysis and their consequences. Such a reflection is essential for the consolidation of disciplinary knowledge, the building of a common language, and the understanding of different methodological decisions.

In summary, we see strong evidence for the usefulness and benefits of network science to support design research and increasing uptake in engineering design studies. However, alongside compelling reasons to conduct network-based analyses, there are also conceptual and methodological challenges. Such challenges need to be understood and addressed in an engineering design field-specific man- ner, so as to conduct rigorous research and to capitalise on the benefits of applying a network-based perspective to the study of the design process. We will sketch the core decision points below and illustrate them with study examples focused on the engineering design process.

The remainder of the chapter is structured as follows: Sect. 9.2 introduces the main methodological challenges and decision points a researcher faces when using network science to support design research. In Sect. 9.3, we provide specific case examples to illustrate core decision points a researcher needs to address and, in particular, we highlight three distinctive characteristics of network approaches: data-driven analyses, a multilevel perspective, and result interpretation facilitated by interactive visualisations. Section 9.4 pinpoints the opportunities for using network science and discusses the core points raised in this chapter, and Sect. 9.5 concludes by providing a summary and outlook.

\subsection{Methodological Challenges and Decision Points}

Well-established and generic methodological guidance in the field of network science exists, including fundamentals on graph theory and network analysis (e.g. Barabási 2012; Diestel 2005). However, despite the emergent use of network analysis in 
engineering design, the field still lacks cohesive field-specific methodological support. Support of the sort that already exists for social sciences such as sociology and organisational studies (e.g. Borgatti et al. 2013; Carrington et al. 2005) and for natural sciences such as physics and biology (e.g. Estrada 2013; Ma'ayan 2012). This support is needed to build and share common terminology and tools, and to further develop research methods that respond to the distinctive characteristics of our field. Some of the characteristics and methodological challenges of engineering design that impact network-based research include the following:

1. The diversity of research questions, units, and levels of analysis. This often requires an understanding of multilevel network analysis methods.

2. The inherent dynamic and socio-technical nature of the design domain. This generates the need for a robust understanding and study of heterogeneous (multimodal and multiplex) dynamic networks that can simultaneously combine multiple elements, including people, activities, documents, and engineering components.

3. The often unknown or unclear direction of causality between network structure and performance. This lack of clarity increases the difficulties of data interpretation, which are in part the results of difficult experimental conditions and the limited possibilities to implement control variables when studying design processes 'in the wild'.

In order to respond to design's distinctive characteristics and methodological chal- lenges when applying network science, we started by examining previous classifica- tions of network studies (e.g. Borgatti and Foster 2003; Parraguez 2015). From those classifications, we can distinguish two key aspects that affect network research within and beyond the design field: (A) the overall research purpose (exploratory and explanatory), including assumptions about the direction of causality (network structure affecting behaviour and performance or vice versa), and (B) network features including main units and levels of analysis. See Fig. 9.1 for an illustration of the key decision points.

\subsubsection{Define Overall Research Purpose}

While design studies often attempt to move from an exploratory and descriptive mode to a hypothesis- and theory-testing one, limitations in available data (sample size, contingency, level of detail, etc.) and the somewhat embryonic state of network theories and methods specific to the design context complicate extrapolation of results. So far, most network studies in design research are primarily exploratory, are theory-generat-ing, and portray case-specific patterns that allow for theory development (e.g. Collins et al. 2010). However, we are also beginning to see examples of network studies mov- ing into explanatory, hypothesis-testing mode (e.g. Sosa et al.2011).

With respect to direction of causality, research in design and most other fields has generally examined how network structure affects behaviour and not vice versa (Borgatti and Halgin 2011). In design research, this can be explained by the fact that most dependent variables are associated with performance measures of sort, either related to the designer (e.g. speed, creativity), the activity (e.g. on time, on budget), 
A. Define the overall research purpose
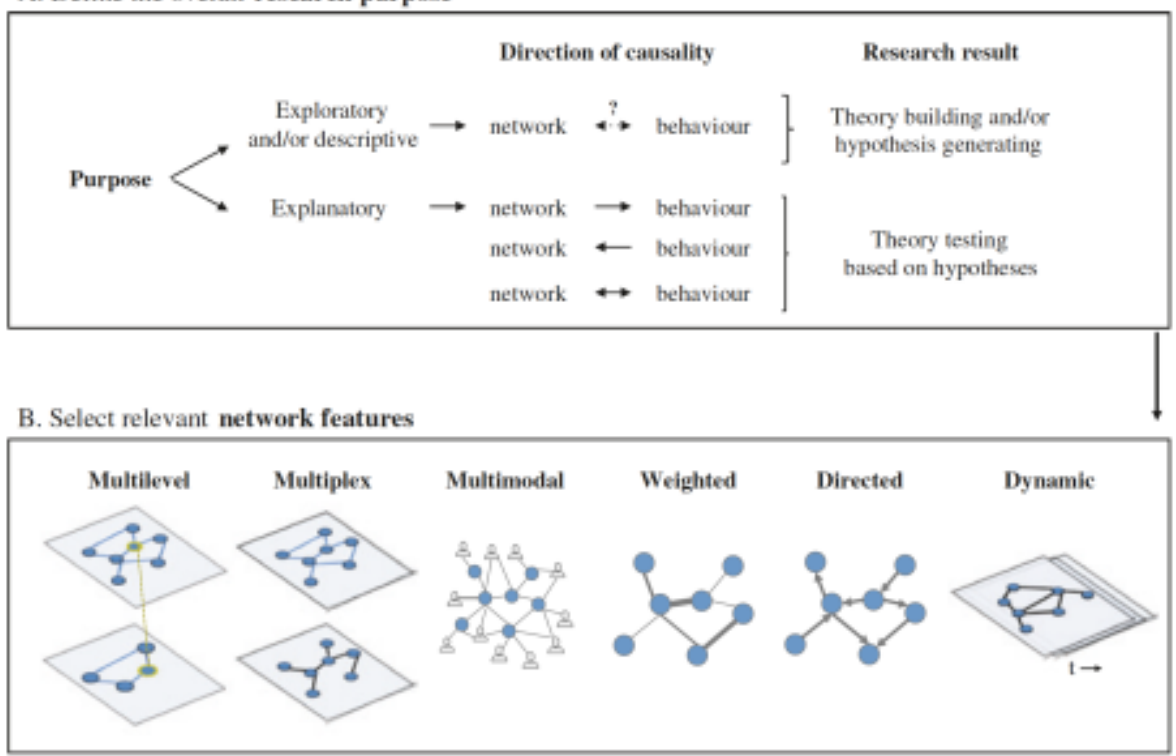

Fig. 9.1 Using network science in design research: decision points. A) Define the overall research purpose. B) Select relevant network features

or to what is being designed (e.g. quality, meeting specifications, novelty). Therefore, network properties and measures (such as network size, density, centrality, and clustering) have mostly been used as predictors or independent variables in the analysis. This reinforces the trend of causality direction of the type 'network structure affects behaviour'. While this logic is sound, it is important to note that in complex systems, causality is rarely unidirectional, and feedback loops are common. For this reason, exploring how the behaviour and attributes of designers and performance outcomes shape the network structure of the design process would also be of value to design research. In summary, if the purpose is exploratory, the causal relationship is not predefined and research is part of theory building and/or hypothesis generation. If the purpose is explanatory, then a causal relationship should be hypothesised upfront and tested.

\subsubsection{Select Relevant Network Features}

Having considered the type of network studies to be undertaken, the researcher wishing to analyse networks needs also to be aware of common network features that affect the methodological design and reach of his/her study. A network conceptualisation of complex systems can be multilevel, multiplex, multimodal, weighted, directed and/or dynamic. However, for analytical reasons and practical purposes, a network may instead be conceptualised and studied from a simpler perspective, i.e. unilevel, non-multiplex, unimodal, unweighted (binary), undirected, and/or static. In any case, we need to address the theoretical and analytical consequences and limitations of selecting certain features and not others. We exemplify 
each feature listed above focusing on the design process. Table 9.1 provides references to generic conceptualisations of each network feature and offers references to the application of each network feature in design-related research.

The units and levels of analysis in network-based design research can vary widely. For example, if the unit of analysis is defined as individual designers, activities, or components, the level of analysis would be nodes in a network. At this node-level, the idea is to quantify the effect of the whole network (or part of it) on each node. In contrast, if the unit of analysis is defined as information exchanges and/or information flows between people or activities, the level of anal-ysis would be edges in a network. At the edge level, the idea is typically to quan- tify the characteristics of each connection between two nodes, e.g. a set of two

Table 9.1 Network features and exemplary references

\begin{tabular}{l|l|l}
\hline $\begin{array}{l}\text { Network feature } \\
\text { Multilevel } \\
\text { also known as } \\
\text { nested networks) }\end{array}$ & $\begin{array}{l}\text { Application in design } \\
\text { research }\end{array}$ \\
\hline $\begin{array}{l}\text { Multiplex } \\
\text { (also known as } \\
\text { multilayer) }\end{array}$ & $\begin{array}{l}\text { The network is studied at } \\
\text { different levels shedding light } \\
\text { on the architecture of nodes, } \\
\text { edges/interfaces and the whole } \\
\text { network or parts of it (e.g. } \\
\text { Brass et al. 2004; Moliterno and } \\
\text { Mahony 2011) }\end{array}$ & $\begin{array}{l}\text { Eppinger et al. } \\
\text { (2014), Johnson } \\
\text { (2005), Parraguez } \\
\text { (2015) }\end{array}$ \\
\hline $\begin{array}{l}\text { Multimodal } \\
\text { (also known as heter- } \\
\text { ogeneous networks) }\end{array}$ & $\begin{array}{l}\text { The same system of } \\
\text { interconnected elements/nodes is } \\
\text { studied through different network } \\
\text { layers. Each layer defines a } \\
\text { different type of relationship } \\
\text { between the elements/nodes (e.g. } \\
\text { Kivelä et al. 2013) }\end{array}$ & $\begin{array}{l}\text { Parraguez (2015), } \\
\text { Pasqual and de Weck } \\
\text { (2011) }\end{array}$ \\
\hline $\begin{array}{l}\text { Weighted } \\
\text { (also known as } \\
\text { valued networks) }\end{array}$ & $\begin{array}{l}\text { The network under study } \\
\text { contains heterogeneous elements/ } \\
\text { nodes (e.g. Wasserman and Faust } \\
\text { 1994: 29) }\end{array}$ & $\begin{array}{l}\text { Durugbo et al. } \\
\text { (2011), Morelli et al. } \\
(1995), \text { Parraguez } \\
\text { (2015) }\end{array}$ \\
\hline $\begin{array}{l}\text { Directed } \\
\text { (also known as } \\
\text { temporal or evolving } \\
\text { networks) }\end{array}$ & $\begin{array}{l}\text { The edges/relations between } \\
\text { elements/nodes are valued to } \\
\text { quantify the strength of the edge/ } \\
\text { relation (e.g. Wasserman and } \\
\text { Faust 1994) }\end{array}$ & $\begin{array}{l}\text { Browning and } \\
\text { Eppinger (2002), } \\
\text { Parraguez and Maier } \\
\text { (2015), Parraguez } \\
\text { (2015), Sosa (2014) }\end{array}$ \\
\hline $\begin{array}{l}\text { The edge/relation between } \\
\text { elements/nodes in the network } \\
\text { has an explicit directionality (e.g. } \\
\text { Wasserman and Faust 1994) }\end{array}$ & $\begin{array}{l}\text { Meier et al. (2007), } \\
\text { Smith and Eppinger } \\
\text { (1997) }\end{array}$ \\
\hline $\begin{array}{l}\text { The elements/nodes and or rela- } \\
\text { tions/edges change over time. } \\
\text { This includes adding, removing } \\
\text { and/or reweighting nodes and/or } \\
\text { edges (e.g. Holme and Saramäki } \\
\text { 2012) }\end{array}$ & $\begin{array}{l}\text { Braha and Bar-Yam } \\
\text { (2007), Collins et al. } \\
\text { (2010), Parraguez } \\
\text { (2015), Parraguez } \\
\text { et al. (2015a) }\end{array}$ \\
\hline
\end{tabular}


(dyads) or three nodes (triads) and their connections. Finally, if the unit of analysis is the whole design process, organisation, or project, the level of analysis would be the whole network or at least subsections thereof.

Multilevel: The network structure of the design process can be analysed from multiple levels, including individual activities (nodes), information flows between activities (edges), and the entire process architecture (whole network). The intrinsically multilevel nature of networks allows design researchers using network science to integrate findings and more fluently move between micro- and macro-levels, maintaining analytical consistency across the examined levels. This is the equivalent of being able to zoom in and zoom out as required by the research question at hand.

Multiplex: A multiplex view of the process architecture means that different types of relationships or interactions between activities are explicitly considered and analysed as different 'network layers'. This could be used to analyse and com- pare the network structure of the actual and planned process under a consistent framework, or to map and compare (actual) information exchanges between people with the (required) information dependencies between tasks.

Multimodal: A multimodal network of the design process would in the same network layer simultaneously include two or more different entities, for example people and activities. While multimodality significantly increases the complexity of the model, it also allows bridging different domains and allows integrating different domains, such as the organisation and process domains.

Weighted: An edge between two nodes in the network may be weighted. This might refer to the intensity or amount of information flowing between two activities. Weighting can be essential to distinguish patterns in the network structure that might otherwise be hidden underneath the homogeneity that a binary (unweighted) relationship between two nodes suggests.

Directed: A relationship between two entities may or may not be directed. That is, energy or material usually flows in a particular direction. Likewise, information exchanges may or may not be reciprocated. As a result, networks are classified as directed or undirected.

Dynamic: The network structure may change over time as nodes and edges appear or disappear over time, due to reweighting of edges and/or changes in relationship types. When analysing a system's structure over long periods of time, the temporal and potentially dynamic evolution of the system can be the key to understanding its structure. This is particularly true when studying complex and evolving processes such as designing, where changes in the emergent behaviours are not only expected but needed to fulfil envisioned objectives.

\subsection{Case Study}

Here, we highlight and exemplify the key decision points detailed previously in this chapter. We illustrate some of the decision points with snapshots from one case example of a renewable energy plant as a large-scale engineering system 
where we used archival data (activity log and email data) spanning three years of design and development.

The focal company has designed, developed, and built steam-generating plants for over 150 years. It has done this in coordination with a partner company and a network of more than 50 external national and international organisations, including a range of suppliers, manufacturers, building contractors, consultants, and regulatory agencies. While their engineering design process works well and complies with the highest industry standards for process and project management, the company has come to realise that to move forward, they require additional support. For example, the complexity of the technology they develop, in conjunction with the fast-paced and competitive market they operate in, has stretched to the limits their organisational set-up and the traditional approaches they use to plan, execute, control, and improve the design process. They have therefore been seeking a systemic overview of their information flow and design activities in order to streamline their process of designing and developing biomass power plants.

For this, they needed a way to view, understand, and monitor their actual design process by way of integrating both the technical (information dependencies between activities in the design process) and social (work-related communications between people in the organisation) dimensions of how they organise their design work. They needed to move beyond counting incidences separately in the prod- uct, process, and organisation domains, to connecting the data, so that they could identify the actual information flows between activities. These flows can only be modelled and understood in the context of information exchanges between people, hence the need for connecting cross-domain data. Further, they needed an objective way of 'measuring' their patterns of information flows.

\subsubsection{Illustration of Methodological Challenges and Decision Points}

(A) Defining the overall research purpose: Due to the company need for a broad and deep overview of their interaction patterns, we set the research purpose as primarily descriptive, seeking to study the actual design process through a systemic, multilevel, socio-technical, and data-driven network approach. We choose not to assume a direction for the causal relationship between the analysed network architectures and observed design process performance. The reason for this was that we needed to first build an appropriate theoretical frame for linking network architecture to performance. Only after the theory- building process was considered appropriate, could we move to an explana- tory, hypothesis-testing mode.

(B) Selecting relevant network features: Our descriptive research purpose required a set of network features consistent with a multilevel socio-technical focus. With these requirements in mind, and given the availability of suitable 
digital data traces, we selected the most comprehensive set of network features possible that were analytically compatible with a study that combines people and activities (process and organisation domains). As a result, this case illustrates the analysis of a wide range of network features through the study of just one design process.

The selected network features included: a multilevel characterisation of the design process to describe the network architecture of design activities, interfaces between activities, and the design process at the whole network level. To study the degree of alignment and influence between the actual and planned design processes, we adopted a multiplex network approach where actual and planned processes represent different network layers which could then be mapped. To cap- ture the socio-technical nature of the design process, we needed to combine peo- ple and activities. To achieve this, we opted for a multimodal network approach, combining the process and organisation domains. To capture the natural spectrum of intensities that occurs in information exchanges between people as well as the variation in the amount of participation in activities, we conducted all our analy- ses using weighted networks. Even though email exchanges show direction, we decided to use undirected instead of directed networks in our analyses, as most email exchanges were reciprocated and affiliation to activities are naturally mod- elled as undirected networks, and because many centrality metrics are not yet refined for directed networks. Finally, we selected a dynamic approach for the level of the whole process in order to explore evolving information centralisation patterns through different stages of the design process.

For more detailed information on specific aspects of this case study, see the following: The overall research approach, named The Networked Process Framework, was developed in Parraguez (2015), the dynamic network analysis of the whole process was published in Parraguez et al. (2014, 2015a), the interface level analysis in Parraguez et al. (2015b), and the activity-level network analysis in Parraguez and Maier (2015).

\subsubsection{Case Snapshots}

The snapshots presented here are the result of applying the proposed network science approach and following the prescribed decision points in our own case study. The objective is to provide an empirical overview and reflection about the outcomes of its application, combining data from the process and organisation domains. These snapshots show the effect of the approach on data acquisition, gathering, and interpretation, highlighting the relevance of network visualisations and the multilevel nature of the analysis. The network analysis was conducted combining network visualisation produced with Gephi (Bastian et al. 2009) and network analyses performed with UCINET (Borgatti et al. 2002). 
Mapping the actual organisation network: Following the tradition of social network analysis and organisational network studies, we modelled the network of actual communications (organisation domain) by mapping the email exchanges between people from the case company as an engineering communication net- work. We drew from a repository that includes more than 10,000 emails between several hundred people in a period of more than three years (September 2009 and August 2013). Figure 9.2a, b shows the analysis including the formal functional affiliation of people within the company. The figures differ in terms of the graphi- cal layout applied to visualise the network and show how different visual represen- tations can lead to different network insights. Figure 9.2a emphasises the natural distribution of people based on their email communications. Figure $9.2 \mathrm{~b}$ arranges the position of each person based on his/her formal functional affiliation, which allows identifying key communication lines between groups.

At this level of detail, we can see individual people inside the focal company, their email communications, and the formal organisational groups they are affili- ated with (shown in node colour and label). This aggregated view of the infor- mation exchanges allows us to identify key players such as the project manager and the leader of on-site integration, and also some unexpectedly central people. Here, it is also possible to analyse the degree of information exchange between the formal organisational groups within the company and other interesting insights for traditional organisational studies. One way of connecting such information exchanges with the design process is by using the simplifying assumption that formal organisational groups can be mapped directly and in a one-to-one fashion onto design activities. However, such an assumption is problematic for complex projects where each activity requires inputs from different technical specialities, and assigning people to activities is more dynamic than assignment of people to formal organisational groups. For example, it is not uncommon for people to be iteratively working on different activities and therefore switching back and forth between activities. Furthermore, our analysis shows that, for this particular case, that assumption would be inadequate because people do not tend to cluster com- munications within their own formal organisational groups. One reason for this is that communication is being clustered around design activities instead of func- tional groups (as Fig. 9.3 reveals).

Mapping of organisation (communications) and process (activities) networks: The bridge between organisation and process was generated by a weighted and multimodal network of people and activities that we built through the use of more than 10,000 activity logs (see Fig. 9.3).

Figure 9.3 shows how people indeed tend to cluster around activities rather than formal functional groups. It also shows the relative diversity of each activ- ity in terms of the number of individuals from different functional groups partici- pating in the activity. The limitation here is that we have no proxy for the direct communication between people, which we know is at least as important as the coparticipation in activities to determine the information flow between activities. To incorporate this, we need to integrate the previous information about email 
(a)

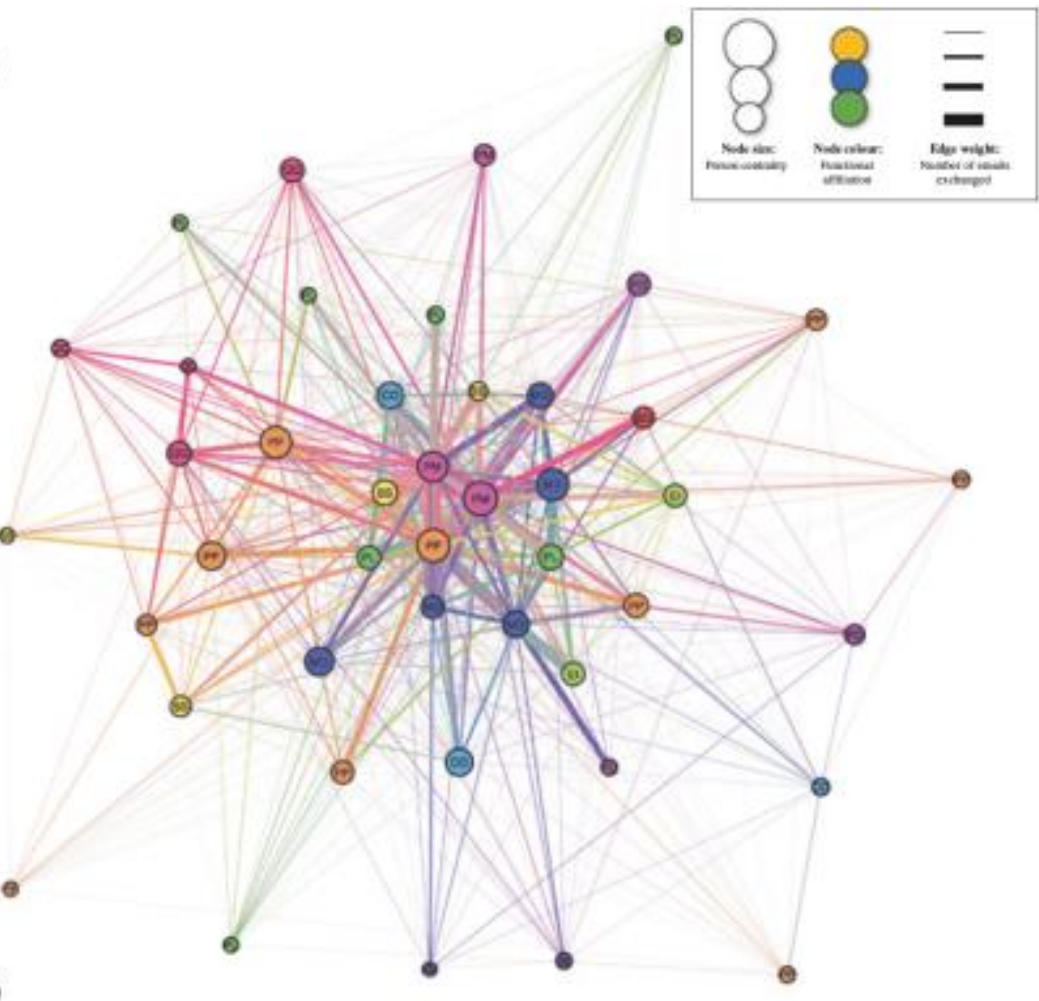

(b)

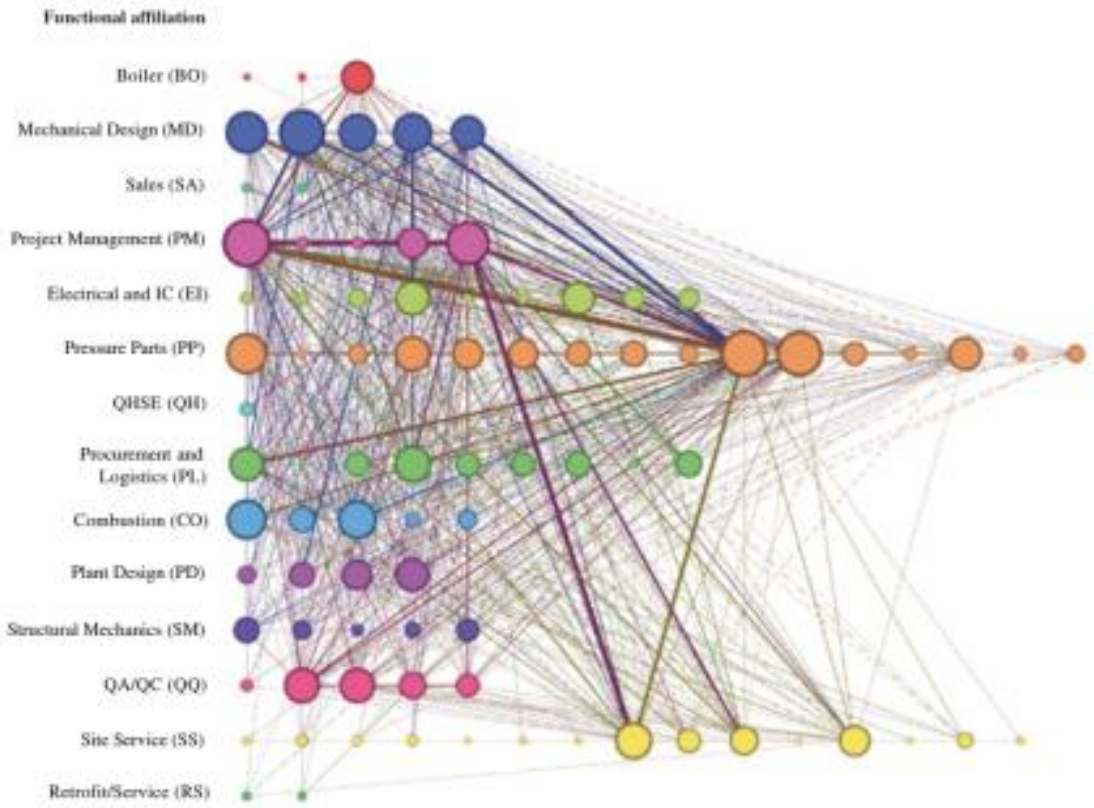

4 Fig. 9.2 a Internal project-level communication. Weighted and undirected simplified network graph showing key people within the design process of the focal company. Force-directed layout highlights the organic distribution of people based on their email communication. Nodes represent people, and edges represent the sum of email exchanges between two individuals. The network analysed includes 85 people and 10,700 emails. Functional affiliations are coloured and labelled, e.g. pressure parts (PP) and project management (PM). See Fig. 9.2b for details. b Internal project-level communication using the same data set as in Fig. 9.2a. Weighted and undirected 
network graph showing key people within the design process of the focal company. Fixed attributional layout highlights organisational affiliation and cross-group communications

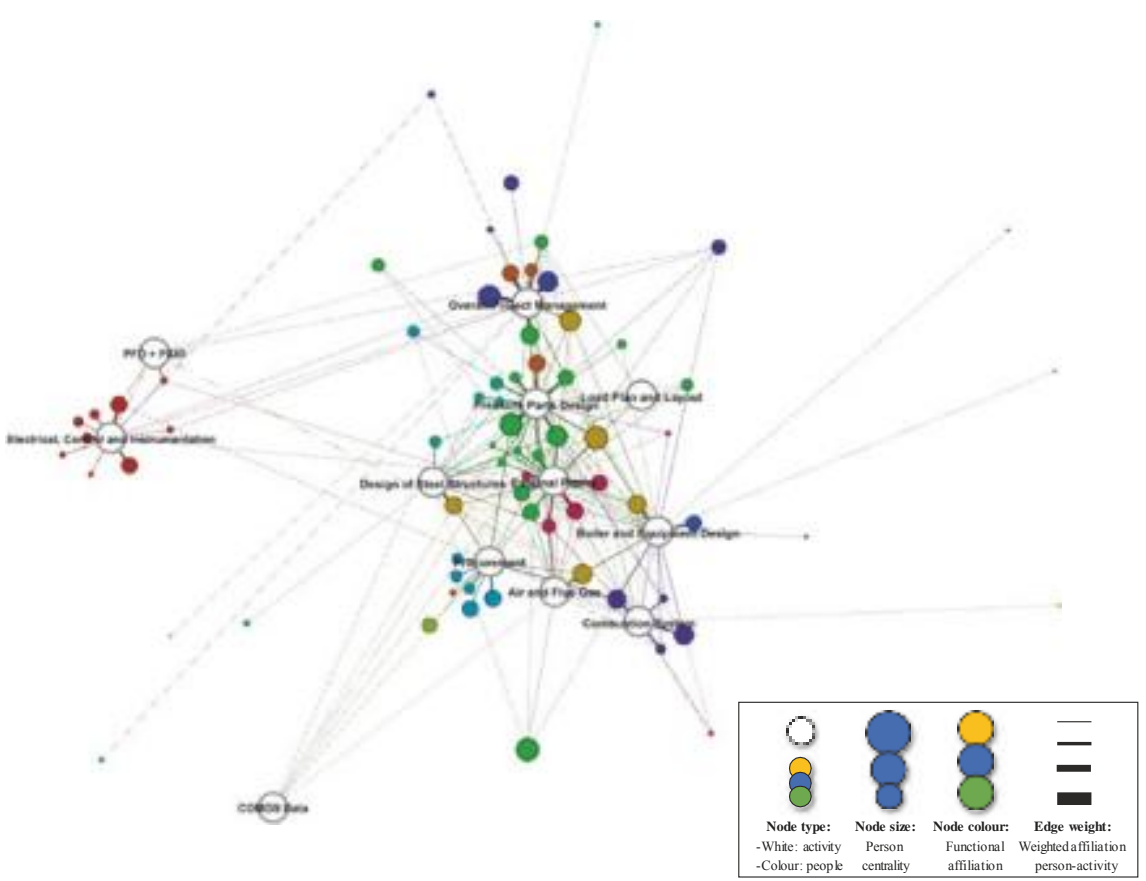

Fig. 9.3 Mapping of 85 people (colour nodes) to 12 activity groups (white nodes) as obtained from archival data. Activity names are based on company codes. Edges show the sum of 10,505 activity records connecting people with activities

exchanges between individuals to the network of people performing activities. Figure 9.4 provides such an integrated view.

Figure 9.4 depicts a compact and rich visualisation of the entire design process in terms of aggregated information flows between activities. Here, we can estimate closeness between activities based on the actual information flow between them, identify central and peripheral activities and people, and calculate a full set of network architecture metrics at different levels of analysis.

Multilevel zooming in and out: In order to exemplify multilevel movements as described above and analogous to zooming in and zooming out in the network, Fig. 9.5 shows a sequence of visualisations that takes us from the whole process level to the activity and interface levels. 


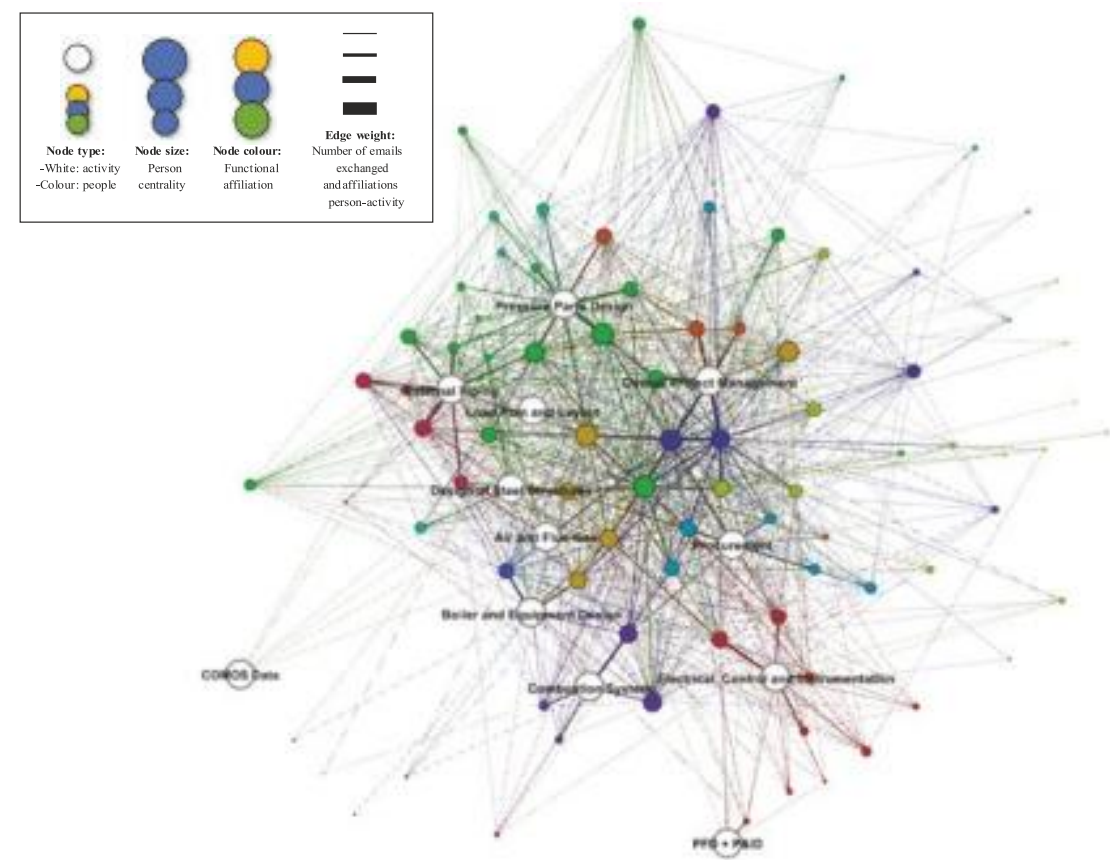

Fig. 9.4 Mapping of people (colour nodes) to activity groups (white nodes) as obtained from archival data. Activity names are based on company codes

Figure 9.5 shows an example of a multilevel network visualisation. In this figure, based on the overall process architecture, we can select two information interdependent activities, for instance, pressure parts design and electrical con-trol and instrumentation. These two design activities are located at opposite ends of the graph and have distinctly different network structures and compositions in terms of network size, diversity, and density. Zooming in from the whole process, in which pressure parts design is embedded, to the level where we can examine pressure parts design as a single activity, we gain an additional level of under- standing with more detail. At the activity level, we can quantify the characteristics of this activity and compare it with other activities in the network. We can also identify specific information roles and subgroups of people, and we can perform a full social network analysis on the engineering communication network associated with the 'inner workings' of this activity. Moving from the study of pressure parts design in isolation to the interface level, and in particular the interface with electri-cal control and instrumentation, we can now observe the joint network that ena- bles the information transformation and information exchange between these two activities. At this interface level, it is possible to quantitatively characterise each interface network as well as to identify key individuals working at each interface. 


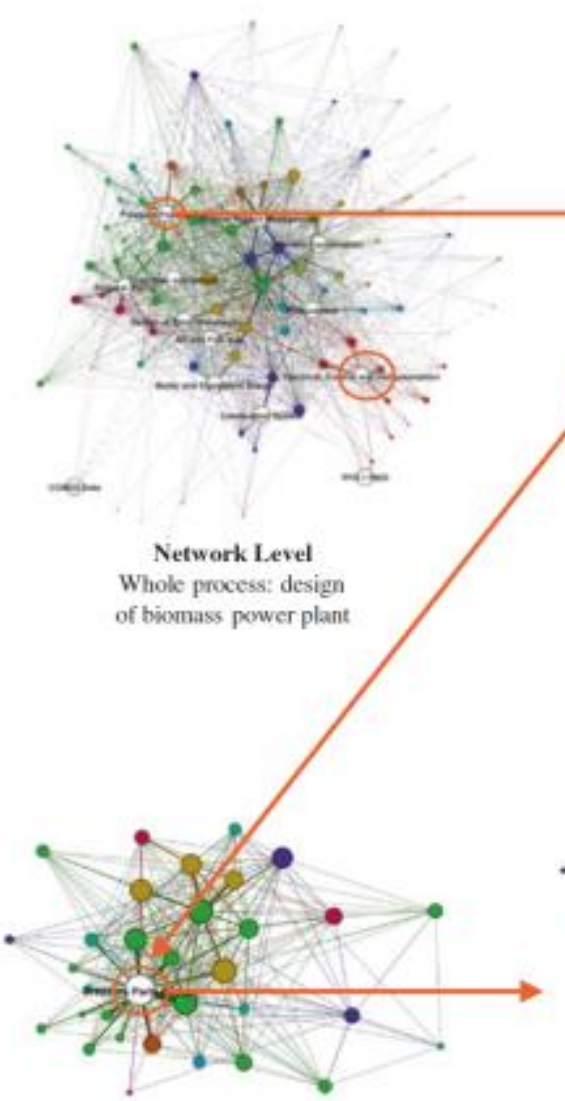

Node Level

Activity: pressure parts design (ego-network)
Network Level

Pressare parts design

embedded in the whole process network

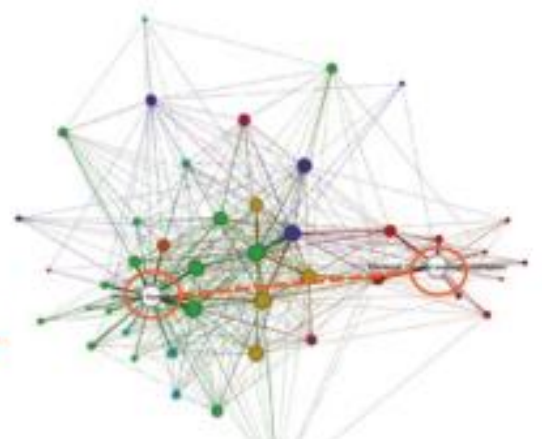

Edge Isvel

Interface: pressure parts-electrical control and instrumentation (joint ego-network)

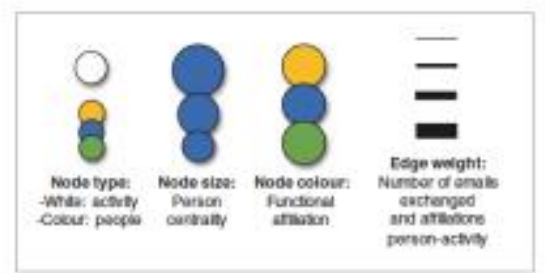

Fig. 9.5 Multilevel network visualisation combining all the previous analysis. Figure illustrates the idea of zooming in and out between network levels 


\subsection{Discussion}

Based on the results of our own studies and previous network science research applied to engineering design (e.g. see Chap. 8), three opportunities for using network science in design research stand out:

(1) Studying the nature of the interactions that occur in the network. This includes how the behaviour of nodes (such as people, activities, or compo- nents) is affected by their location in the network; or how the qualities or attributes of the nodes (e.g. functional affiliation to departments, activities types, or categories) influence the structure of the network.

(2) Studying how network structure (e.g. centrality, connectivity, cluster- ing, degree distribution, density, path length, size) influences processes that develop over the network, e.g. spread or propagation effects, such as spread of information or the propagation of changes; with the core idea of how net- work structure influences a (stochastic) process of spread. For example, the way in which information flows through design activities and is exchanged between designers in the organisation, or the propagation of changes and errors in the design process.

(3) Knowledge of network properties allows us to use network models to test the effect of interventions. One of the core motivations for developing network models is to come up with a virtual laboratory representing (socio-techni- cal) systems with (mathematical) representations, for example, to generate answers to what-if scenarios. To illustrate, we may test for resilience, robust- ness, and vulnerability of the design process and design organisation when facing disruptions such as the loss of key designers or staff changes following an organisational restructuring. More generally, we may probe for patterns of how relationships or information spread, or how we may encourage or inhibit the spread of information.

It is often argued that an holistic and systemic perspective is necessary to understand patterns of designing (Eckert et al. 2005). This requires moving between qualitative and quantitative research modes as well as between detailed microanalyses of design activities and designer behaviour and macro-analyses of the whole design process.

Network-based research, due to its inherently multilevel and system-oriented nature, plays a key role in responding to this need. Through the snapshots of our case study, we have shown how applied network science may be used to integrate large amounts of process data with qualitative case study interpretations, providing a quantitative multilevel platform from which to examine the design process and from which to gain new insights. Through such a network perspective, we can connect otherwise disconnected data and generate knowledge ranging from individual activities and people to whole process-level dynamics. We thereby facilitate connecting the dots between what design researchers have learned through in-depth studies at different levels of the design process. Furthermore, researchers exploring computational modelling of teamwork in design (see Chap. 10) and other types 
of computer simulations (see Chap. 11) can use the empirical results of network analyses to support modelling and parameterisation of their computer models. We thereby gain precision and allow for the integration of empirical data produced by other researchers. In the same way, design researchers working with qualita- tive case studies can complement their analyses and interpretations of the case with quantitative analyses and visualisations produced by network-based approaches.

\subsection{Conclusions}

We have in the last three decades seen a growing number of studies applying dif- ferent variants of network analyses to the design process. However, despite this increasing interest, so far there has been little convergence and reflection about the challenges, methods, and key decisions surrounding the implementation of network- based research in design. Furthermore, as Cash and Culley (2014) write on 'the role of experimental studies in design research', so too do we take liberty to say that there is a lack of field-specific guidance for using network approaches in design research.

In this chapter, we contribute to this field-specific guidance providing a summary of core decision points that design researchers can use as a reference when planning and implementing their studies. We illustrate these decision points through a case study, examining the structure and dynamics of a real engineering design process. Our emphasis is on multilevel analyses and on the importance of visualisations. These visualisations serve as tools for eliciting (qualitative) feedback, for validating results, and for collaboratively interpreting findings, e.g. in a case study setting.

With this contribution, we aim to facilitate the fruitful integration of network science into the toolbox of both qualitative and quantitative design researchers, in particular those with an interest in multilevel design research. Similarly, and in connection with the next chapter in this book on simulations, the results of empirical network analyses of the design process provide key inputs for the development of more detailed simulation-based approaches of designing. Such inputs provide researchers using simulations with quantitative parameters and process topologies that feed their models and test their simulations.

As noted in the chapter, there are many significant works on applied network science. It is hoped that design researchers also turn to these works for inspira- tion and further guidance on ways of using network analysis to support and further design research and design management practice.

Encouraged by several discussions with colleagues in the growing field of network science, we would also like to point out that design practice provides a unique and wonderfully rich socio-technical fabric of interactions that research- ers from other fields are eager to get their hands on and heads around. This offers interesting interdisciplinary research opportunities for mapping, understanding, and predicting relationships between network architecture, observed socio-technical behaviours, and performance. 


\section{References}

Ahn Y-Y, Bagrow JP, Lehmann S (2010) Link communities reveal multiscale complexity in networks. Nature 466:761-765

Albert R, Barabási A-L (2002) Statistical mechanics of complex networks. Rev Mod Phys 74:47

Allen TJ (1977) Managing the flow of technology: technology transfer and the dissemination of technological information within the $r \& d$ organization. The MIT Press

Baldwin C, MacCormack A, Rusnak J (2013) Hidden structure: using network methods to map system architecture (No. 13-093). Harvard Business School

Barabási A (2012) Network science. BarabasiLab, Boston

Bastian M, Heymann S, Jacomy M (2009) Gephi: an open source software for exploring and manipulating networks. In: International AAAI conference on weblogs and social media

Batallas DA, Yassine AA (2006) Information leaders in product development organizational networks: social network analysis of the design structure matrix. IEEE Trans Eng Manag 53:570-582

Borgatti S, Foster P (2003) The network paradigm in organizational research: a review and typology. J Manage 29:991-1013

Borgatti S, Halgin DS (2011) On network theory. Organ Sci 22:1168-1181

Borgatti S, Everett MG, Freeman LC (2002) Ucinet for windows: software for social network analysis

Borgatti S, Everett MG, Johnson JC (2013) Analyzing social networks. SAGE Publications, London

Braha D, Bar-Yam Y (2007) The statistical mechanics of complex product development: empirical and analytical results. Manag Sci 53:1127-1145

Brass DJ, Galaskiewicz J, Greve HR, Tsai W (2004) Taking stock of networks and organizations: a multilevel perspective. Acad Manag J 47:795-817

Browning TR, Eppinger SD (2002) Modeling impacts of process architecture on cost and schedule risk in product development. IEEE Trans Eng Manag 49:428-442

Calvano CN, John P (2004) Systems engineering in an age of complexity. Syst Eng 7:25-34

Carrington PJ, Scott J, Wasserman S (2005) Models and methods in social network analysis. Cambridge University Press, Cambridge

Cash P, Culley S (2014) The role of experimental studies in design research. In: Rodgers P, Yee J (eds) Routledge companion to design research. Routledge, pp 175-189

Christakis N, Fowler JH (2011) Connected: the surprising power of our social networks and how they shape our lives. Back Bay Books, New York

Collins ST, Bradley J, Yassine AA (2010) Analyzing product development task networks to examine organizational change. IEEE Trans Eng Manag 57:513-525

Diestel R (2005) Graph theory. Springer, Berlin [etc.]

Durugbo C, Hutabarat W, Tiwari A, Alcock JR (2011) Modelling collaboration using complex networks. Inf Sci (Ny) 181:3143-3161

Eckert CM, Maier AM, McMahon C (2005) Communication in design. In: Clarkson J, Eckert C (eds) Design process improvement: a review of current practice. Springer, Cambridge, pp 233-261

Eppinger SD, Browning TR (2012) Design structure matrix methods and applications. The MIT Press, Cambridge

Eppinger SD, Salminen V (2001) Patterns of product development interactions. In: Proceedings of the 13th international conference on engineering design (ICED 2001). Glasgow

Eppinger SD, Joglekar NR, Olechowski A, Teo T (2014) Improving the systems engineering process with multilevel analysis of interactions. Artif Intell Eng Des Anal Manuf 28:323-337

Estrada E (2013) Graph and network theory in physics. University of Strathclyde, Department of Mathematics and Statistics, Glasgow

Giffin M, de Weck OL, Bounova G, Keller R, Eckert C, Clarkson PJ (2009) Change propagation analysis in complex technical systems. J Mech Des 131:081001 
Gokpinar B, Hopp W, Iravani S (2010) The impact of misalignment of organizational structure and product architecture on quality in complex product development. Manag Sci 56:468-484

Holme P, Saramäki J (2012) Temporal networks. Phys Rep 519:97-125

Johnson J (2005) Complexity science in collaborative design. CoDesign 1:223-242

Kivelä M, Arenas A, Barthelemy M, Gleeson JP, Moreno Y, Porter MA (2013) Multilayer networks (physics and society). arXiv, eprint arXiv:1309.7233. arXiv

Kreimeyer M, Lindemann U (2011) Complexity metrics in engineering design: managing the structure of design processes. Springer, London

Lindemann U, Maurer MS, Braun T (2009) Structural complexity management. Springer, Berlin Ma'ayan A (2012) New frontiers of network analysis in systems biology. Springer, Dordrecht

Maier AM, Kreimeyer M, Hepperle C, Eckert CM, Lindemann U, Clarkson PJ (2008) Exploration of correlations between factors influencing communication in complex product development. Concurr Eng 16:37-59

Meier C, Yassine AA, Browning TR (2007) Design process sequencing with competent genetic algorithms. J Mech Des 129:566-585

Moliterno TP, Mahony DM (2011) Network theory of organization: a multilevel approach. J Manag 37:443-467

Morelli MD, Eppinger SD, Gulati RK (1995) Predicting technical communication in product development organizations. IEEE Trans. Eng. Manag 42:215-222

Newman MEJ (2003) The structure and function of complex networks. SIAM Rev 45:167-256

Parraguez P (2015) A networked perspective on the engineering design process: at the intersection of process and organisation architectures. $\mathrm{PhD}$ Thesis, Technical University of Denmark

Parraguez P, Maier AM (2015) Unfolding the design process architecture: a networked perspective on activities. In: International conference on engineering design 2015 (ICED 2015), Milan

Parraguez P, Eppinger SD, Maier AM (2014) Evolution of information control and centralisa- tion through stages of complex engineering design projects. In: Dorian M, Mario S, Neven P, Nenad B (eds) Proceedings of DESIGN 2014, the 13th international design conference, Dubrovnik

Parraguez P, Eppinger SD, Maier AM (2015a) Information flow through stages of complex engineering design projects: a dynamic network analysis approach. IEEE Trans Eng Manag

Parraguez P, Eppinger SD, Maier AM (2015b) Characterising design process interfaces as organisation networks: insights for engineering systems management. Syst Eng (in press)

Pasqual MC, de Weck O (2011) Multilayer network model for analysis and management of change propagation. Res Eng Des 23:305-328

Sharman DM, Yassine AA (2004) Characterizing complex product architectures. Syst Eng 7:3560

Simon HA (1962) The architecture of complexity. Proc Am Philos Soc 106:467-482

Smith R, Eppinger SD (1997) A predictive model of sequential iteration in engineering design. Manag Sci 43:1104-1120

Sonnenwald DH (1996) Communication roles that support collaboration during the design process. Des Stud 17:277-301

Sosa ME (2010) Where do creative interactions come from? The role of tie content and social networks. Organ Sci 22:1-21

Sosa ME (2014) Realizing the need for rework: from task interdependence to social networks. Prod Oper Manag 23:1312-1331

Sosa ME, Eppinger SD, Rowles CM (2007) A network approach to define modularity of components in complex products. J Mech Des 129:1118

Sosa ME, Mihm J, Browning TR (2011) Degree distribution and quality in complex engineered systems. J Mech Des 133

Steward DV (1981) The design structure system: a method for managing the design of complex systems. IEEE Trans Eng Manag 28:71-74 
Storga M, Stankovic T, Cash P, McAloone TC (2013) Visually augmented analysis of sociotechnical networks in engineering systems design research. In: Proceedings of TFTD13, Luxembourg

Strogatz SH (2001) Exploring complex networks. Nature 410:268-276

Vespignani A (2009) Predicting the behavior of techno-social systems. Science (80-.) 325:425428

Wasserman S, Faust K (1994) Social network analysis: methods and applications. Cambridge University Press, Cambridge

Wynn DC, Caldwell NH, Clarkson PJ (2014) Predicting change propagation in complex design workflows. J. Mech, Des 136 